OPEN ACCESS

Edited by:

Monica Livia Gheorghiu, Carol Davila University of Medicine and

Pharmacy, Romania

Reviewed by: Lucio Vilar,

Federal University of Pernambuco, Brazil

Andrzej Lewinski,

Medical University of Lodz, Poland

${ }^{*}$ Correspondence:

Małgorzata Rolla

malgorzata.rolla@

student.umed.wroc.pl

Specialty section:

This article was submitted to

Pituitary Endocrinology,

a section of the journal

Frontiers in Endocrinology

Received: 15 December 2020

Accepted: 16 February 2021

Published: 16 March 2021

Citation:

Rolla M, Jawiarczyk-Przybyłowska A

Halupczok-Żyła J, Kałużny M, Konopka BM, Błoniecka I, Zieliński G and Bolanowski M (2021)

Complications and Comorbidities of Acromegaly - Retrospective Study in Polish Center.

Front. Endocrinol. 12:642131. doi: 10.3389/fendo.2021.642131

\section{Complications and Comorbidities of Acromegaly-Retrospective Study in Polish Center}

\author{
Małgorzata Rolla ${ }^{1 *}$, Aleksandra Jawiarczyk-Przybyłowska ${ }^{1}$, Jowita Halupczok-Żyła ${ }^{1}$, \\ Marcin Kałużny ${ }^{1}$, Bogumil M. Konopka ${ }^{2}$, Izabela Błoniecka ${ }^{3}$, Grzegorz Zieliński ${ }^{4}$ \\ and Marek Bolanowski ${ }^{1}$ \\ ${ }^{1}$ Department of Endocrinology, Diabetes and Isotope Therapy, Wroclaw Medical University, Wroctaw, Poland, ${ }^{2}$ Department \\ of Biomedical Engineering, Faculty of Fundamental Problems of Technology, Wroclaw University of Science and Technology, \\ Wroctaw, Poland, ${ }^{3}$ Department of Endocrinology, Diabetes and Isotope Therapy, University Clinical Hospital, Wrocław, \\ Poland, ${ }^{4}$ Department of Neurosurgery, Military Institute of Medicine, Warsaw, Poland
}

Introduction: In acromegaly, chronic exposure to impaired GH and IGF-I levels leads to the development of typical acromegaly symptoms, and multiple systemic complications as cardiovascular, metabolic, respiratory, endocrine, and bone disorders. Acromegaly comorbidities contribute to decreased life quality and premature mortality. The aim of our study was to assess the frequency of acromegaly complications and to evaluate diagnostic methods performed toward recognition of them.

Materials and Methods: It was a retrospective study and we analyzed data of 179 patients hospitalized in the Department of Endocrinology, Diabetes and Isotope Therapy in Wroclaw Medical University (Poland) in 1976 to 2018 to create a database for statistical analysis.

Results: The study group comprised of 119 women (66\%) and 60 men (34\%). The median age of acromegaly diagnosis was 50.5 years old for women (age range 20-78) and 46 for men (range 24-76). Metabolic disorders (hyperlipidemia, diabetes, and prediabetes) were the most frequently diagnosed complications in our study, followed by cardiovascular diseases and endocrine disorders (goiter, pituitary insufficiency, osteoporosis). BP measurement, ECG, lipid profile, fasting glucose or OGTT were performed the most often, while colonoscopy and echocardiogram were the least frequent.

Conclusions: In our population we observed female predominance. We revealed a decrease in the number of patients with active acromegaly and an increase in the number of well-controlled patients. More than $50 \%$ of patients demonstrated a coexistence of cardiac, metabolic and endocrine disturbances and only $5 \%$ of patients did not suffer from any disease from those main groups.

Keywords: acromegaly, complication, comorbidity, pituitary adenoma, IGF-I, GH 


\section{INTRODUCTION}

Acromegaly is a rare endocrine disease associated with elevated growth hormone (GH) and insulin-like factor I (IGF-I) levels, mainly due to a pituitary adenoma (1). Hypersecretion of GH and IGF-I leads to increased cellular proliferation and differentiation, followed by remodelling of tissues, organ enlargement (organomegaly), and disturbances to metabolism. Most acromegaly patients experience a long delay between the appearance of the first symptoms of the disease, its diagnosis, and the start of the treatment (2). In consequence, there is a chronic exposure to increased GH and IGF-I levels leading to the development of typical acromegaly symptoms, and multiple systemic complications as cardiovascular, metabolic, respiratory, endocrine, and bone disorders $(3,4)$. Cardiovascular disorders leading to myocardial infarction and stroke, respiratory diseases, and cancers are the main reasons for premature mortality in this group $(5,6)$. Early diagnosis of acromegaly and its treatment is mandatory to avoid comorbid diseases and further complications leading to premature death. Radical surgery as well as pharmacological control of disease activity decrease mortality to that observed in the normal population $(7,8)$. Moreover, a lot of acromegaly patients suffer from decreased life quality despite cured or well-controlled disease activity $(9,10)$. Complications like diabetes, cardiovascular disease and vertebral fractures contribute to a significant reduction of patients' quality of life $(11,12)$.

First guidelines about diagnosis and treatment of acromegaly complications were published in 2003 (3). Since then some updates emerged, with the most relevant in 2013 (13) and 2020 (14), recommending a change in approach to the disease supporting holistic view on an acromegaly patient with involvement of different types of specialist. As stated in screening for comorbidities should be performed at the time of diagnosis and repeated regularly.

The aim of our study was to perform an in-depth analysis of the gathered database of hospitalization in terms of acromegaly complication frequencies and their co-occurrence. Also, we investigated the changes in procedures used in diagnostics and treatment of acromegaly patients over an eighteen-year period, from 2000 to 2018 .

\section{MATERIALS AND METHODS}

In this retrospective study, we analyzed data of 179 patients hospitalized in the Department of Endocrinology, Diabetes and Isotope Therapy in Wroclaw Medical University (Poland) in 1976 to 2018 . The inclusion criteria were diagnosis of acromegaly according to Endocrine Society Guidelines (15) - elevated IGF-I levels and unsuppressed GH in OGTT at present or in the past. The study included patients with an established acromegaly diagnosis. The database of the patients was created by a single researcher and the registry included 560 records. Data came from patients' hospitalizations at the Department of Endocrinology, Diabetes and Isotope Therapy in Wroclaw and the Neurosurgery
Department in Warsaw. Registry included patients' demographic characteristics, laboratory test results and performed procedures, as well as recommendations for the patients. The number of hospitalizations for each patient was between 1 and 16, with a mean of 3.136 for women and 3.151 for men. The average time of monitoring was 5 years.

Statistical analysis was performed using $\mathrm{R}$ for Windows, version 3.5.3.

\section{Occurrence of Micro- and Macroadenomas}

For 132 patients adenoma sizes were available. An adenoma was classified as a macroadenoma if at least one of its sizes was greater than $10 \mathrm{~mm}$. The comparison of micro- to macroadenomas between males and females was performed using the Chi-squared test.

\section{Comparison of Patients Who Underwent One vs. Several Surgeries}

All comparisons were performed separately for male and female patients. The IGF-I concentrations and GH concentrations were compared using the Wilcoxon Rank Sum test. GH concentrations were log-transformed prior to analysis. The comparison was also performed with Student's t-test (data not shown) - with consistent results. The comparison of complications occurrence (pituitary insufficiency, secondary hypogonadism, panhypopituitarism) between the groups was performed using the Chi-squared test.

\section{Comparison of Complication Frequencies Between Male and Female Patients}

The comparison for each complication was performed using the Chi-squared test with Benjamin-Hochberg False Discovery Rate correction $(\mathrm{BH})$.

\section{Analyzing Complications Occurrence With Respect to Patient Age}

The analysis was performed separately for male and female patients. For each patient, we have found at what age the analyzed complication first occurred. Age values were categorized into 10-year ranges starting $(0,30],(30,40]$ and so on till $(80,100]$. The bins were then compared using Chi-squared with $\mathrm{BH}$ correction to see if the occurrence is related to patient age.

\section{Clustering Analysis of Complication's Co-Occurrences}

The heatmap was generated by performing agglomerative clustering first, to group patients that suffered from similar complications (this provided the patient clustering dendrogram), second, to group co-occurring complications (this provided the complications clustering dendrogram). In both cases, the Ward's clustering algorithm was used (16) with binary distance metric.

P-value less than 0.05 was considered statistically significant. The study was approved by the local ethical committee. 


\section{RESULTS}

Among the group, there were 119 women (66\%) and 60 men (34\%). The median age of acromegaly diagnosis was 50.5 years old for women (age range, 20-78) and 46 for men (range, 24-76). During the last registered hospitalization, among patients with known disease status, 69 patients (39.7\%) had cured acromegaly, $52(29.9 \%)$ had still active disease and $53(30.5 \%)$ were pharmacologically well-controlled. Over the years the proportion between active acromegaly patients and wellcontrolled patients decreased (Figure 1). In 5-year periods, analyzed from 2000 to 2018, there is a statistically significant decreasing trend in the number of active disease patients ( 80 vs. 29,2000 to 2005 vs. after 2015 , respectively; $\mathrm{p}=0.01$ ), this is accompanied by an increasing fraction of well-controlled acromegaly patients - the trend of percentage of WCA cases was significantly increasing $(7.1 \%$ vs. $43.5 \%, \mathrm{p}=0.02)$.

In 132 patients, there was information about tumor size. Among patients with active acromegaly (102 patients), there was the predominance of macroadenomas $(n=78,76.5 \%)$. In this group, we found a higher ratio of incidence of macroadenomas to microadenomas in men (31/7) than in women (47/17), however, this difference was not statistically significant $(p=0.49)$. In the tumor sizes analysis, we have found statistically significant correlations with IGF-I $(\mathrm{p}<0.000001)$ and GH levels $(\mathrm{p}<0.000001)(\mathrm{GH}$ levels were log-transformed prior analysis for details see Methods section) in the AA group.

139 patients underwent surgery: 110 were operated on once, 20 twice, 6 patients had three, and 3 had four surgeries. 134 patients had transsphenoidal and 10 transcranial surgeries. In 5 cases both types of operation were performed. 108 patients were operated once transsphenoidal, at 26 cases reoperations by the same method were performed.

GH concentrations and tumor sizes (maximal tumor dimensions) were significantly higher among males with more than one surgery compared to operated only once ( $\mathrm{p}=0.00006$; $\mathrm{p}=0.04$, respectively). This difference was not observed among females. Interestingly, IGF-I concentrations were significantly lower among females, but not among males, operated more than once in comparison to females with one surgery $(\mathrm{p}=0.01)$. These results are presented in Figure 2.

In most cases (98/179 - 54.7\%) combination therapy was performed. The most often chosen option of treatment was a combination of surgical and pharmacological therapy (in $45.3 \%$, $81 / 179$ ). Combination of surgery with radiotherapy was performed in $2.2 \%(4 / 179)$ and pharmacotherapy with radiotherapy in $1.1 \%(2 / 179)$. In $6.1 \%(11 / 179)$ combination of all the three methods were applied. In some cases, only one method of treatment was applied - surgery in 24.6\% (44/179) and pharmacotherapy in $13.4 \%(24 / 179)$.

The type of pharmacological therapy used in patients underwent changes throughout the years. Sparsely available information regarding therapy used before 2000 indicates that most of the patients were treated with dopamine agonists (Figure 3). After 2000, somatostatin analogues became a dominant type of pharmacotherapy. Among them, up to 2015 octreotide LAR was preferred, and after 2015 - lanreotide Autogel became the most often chosen. Pasireotide LAR is used in few patients in recent years while pegvisomant was introduced in Poland later, in 2019.

\section{Complications of Acromegaly}

Metabolic disorders (hyperlipidemia, diabetes, and prediabetes) were the most frequently diagnosed complications in our study, followed by cardiovascular diseases and endocrine disorders (goiter, pituitary insufficiency, osteoporosis). More detailed data about the frequency of occurrence of specific complications and their distribution in males and females are presented in Table $\mathbf{1 .}$

Hyperlipidemia was the most frequent comorbid disease and metabolic disorder (74\%). We analyzed the lipid profiles from the first hospitalizations of the patients with hyperlipidemia. In 126/ 133 cases we had complete data about cholesterol and triglycerides levels. 56 patients $(44 \%)$ had high cholesterol $(>200 \mathrm{mg} / \mathrm{dL})$ with normal triglycerides levels $(\leq 150 \mathrm{mg} / \mathrm{dL}) .20$ patients $(16 \%)$ had isolated hypertriglyceridemia, whereas the coincidence of
A

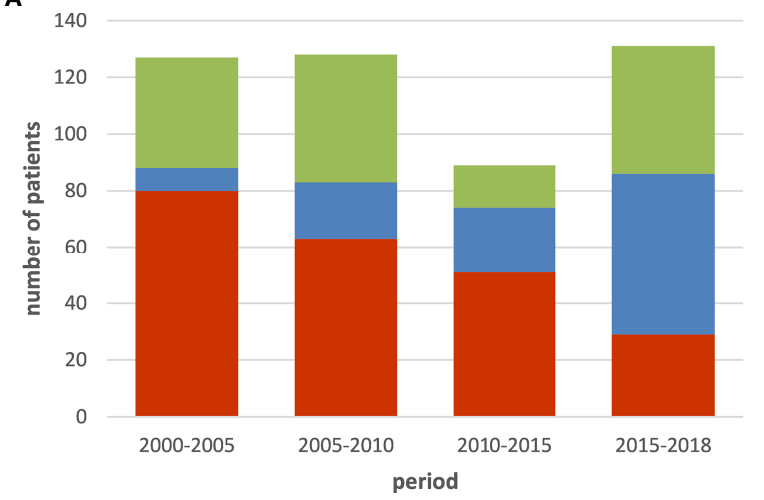

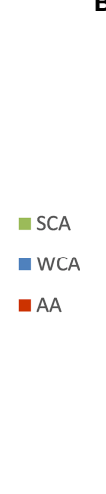

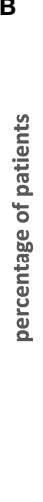

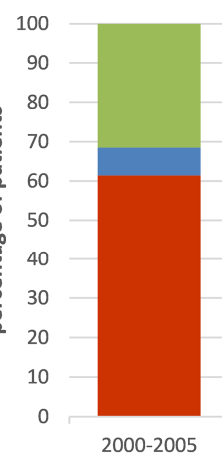
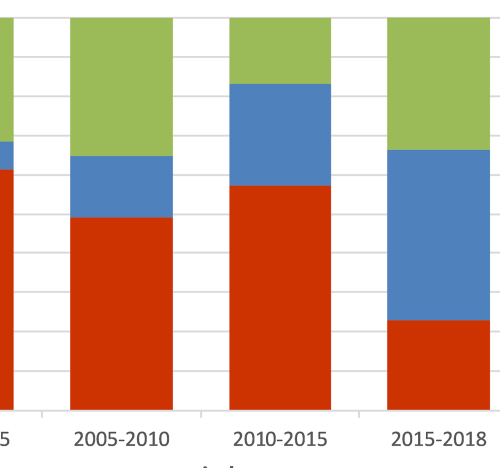

period

FIGURE 1 | Distribution of patients with active, controlled and cured acromegaly over 5-year time frames. (A) - the numerical value; (B) - the percentage value AA, active acromegaly; WCA, well-controlled acromegaly; SCA, surgery cured acromegaly. 


\section{A}
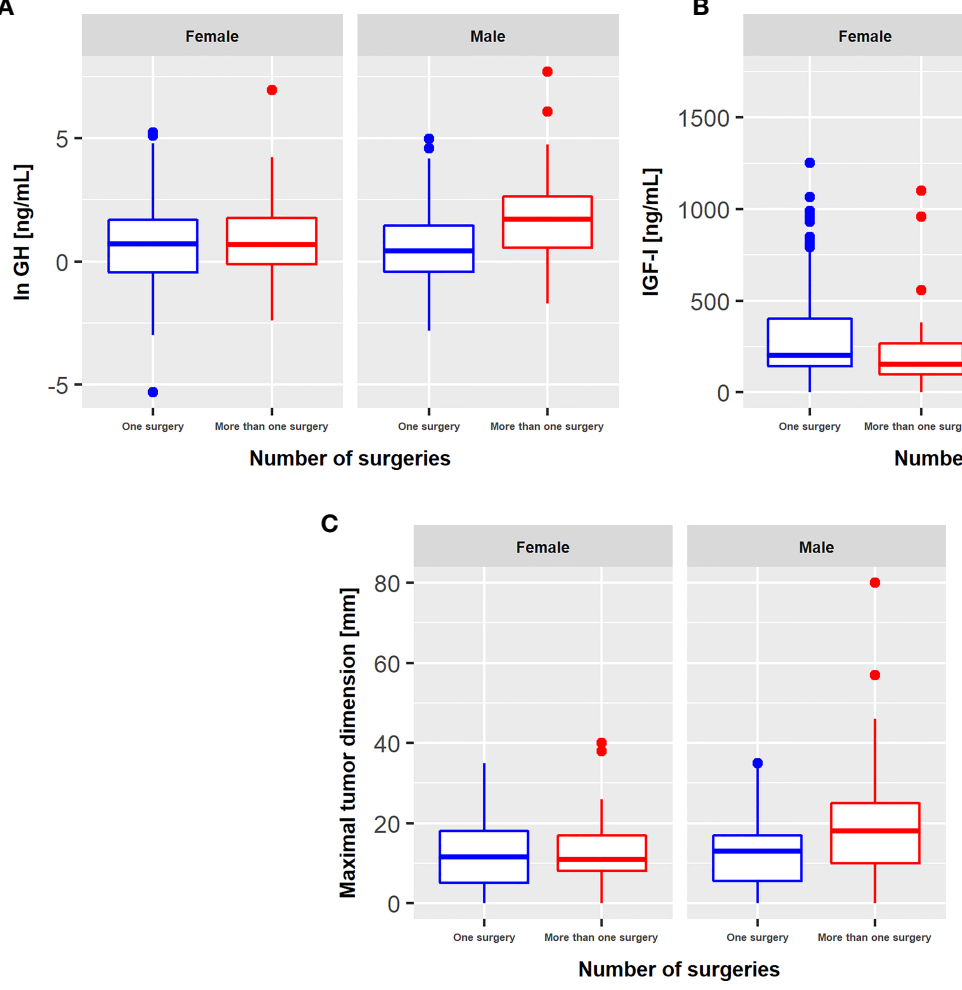

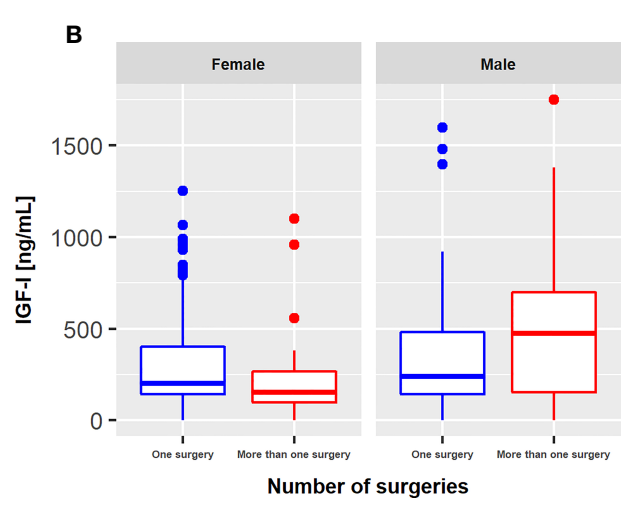

FIGURE 2 | Differences between patients operated once and patients with reoperations. (A) GH concentrations; (B) IGF-I concentrations; (C) maximal tumor dimensions.

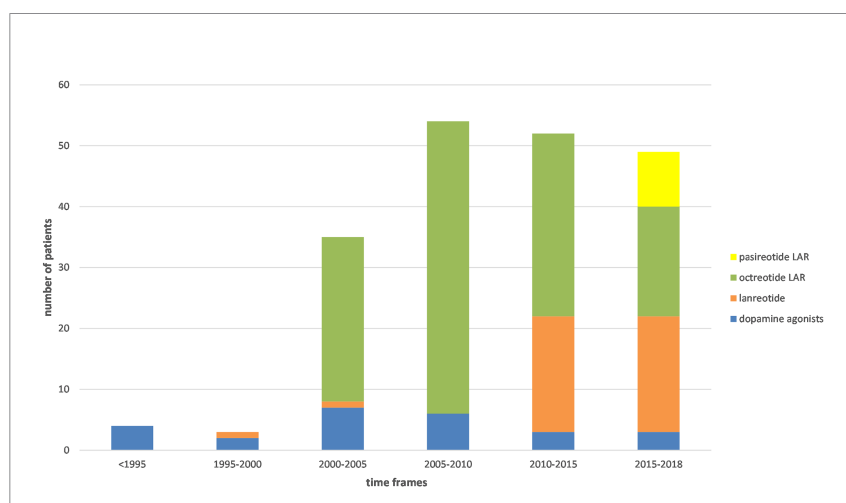

FIGURE 3 | Type of used pharmacotherapy over 5-year time frames.

hypercholesterolemia and hypertriglyceridemia was observed in 40 cases (32\%). In 19 patients coexistence of hypertriglyceridemia with low levels of $\mathrm{HDL}$ ( $<40 \mathrm{mg} / \mathrm{dL}$ in men and $<45 \mathrm{mg} / \mathrm{dL}$ in women) was observed, however in 44 cases there is a lack of information about HDL results. Among cardiovascular diseases, hypertension occurred the most often (58\%), and among endocrine - goiter (52\%). Hyperlipidemia (90/119), hypertension (72/119), and goiter $(65 / 119)$ were the most frequent complications in female patients. All the three occurred in more than half of the subgroup. In the male patients hyperlipidemia also dominated (43/60), hypertension was at the second place $(31 / 60)$, but at third, there was hypopituitarism (30/60). Similarly, all of the three were observed in at least half of the subgroup.

Pituitary insufficiency (insufficiency of one pituitary axis), secondary hypogonadism, panhypopituitarism, and prediabetes occurred more frequently in males than females $(p=0.01$; $\mathrm{p}<0.0005 ; \mathrm{p}=0.003 ; \mathrm{p}=0.01$, respectively). The analysis showed that in males pituitary insufficiency, as well as secondary hypogonadism was more common in patients after radiotherapy ( $\mathrm{p}=0.04 ; \mathrm{p}=0.008$, respectively). Radiotherapy did not have a significant effect on the incidence of panhypopituitarism $(\mathrm{p}=0.09)$. The size of tumor did not have any impact on the incidence of pituitary insufficiency, secondary hypogonadism, panhypopituitarism $(\mathrm{p}=0.23 ; \mathrm{p}=0.52 ; \mathrm{p}=0.21$, respectively). Among males and females with more than one surgery, pituitary insufficiency, secondary hypogonadism, and panhypopituitarism were observed more frequently compared to males and females with one operation (for males $\mathrm{p}=0.001 ; \mathrm{p}=0.002 ; \mathrm{p}=0.009$, respectively, for females $\mathrm{p}=0.0001 ; \mathrm{p}=0.04 ; \mathrm{p}=0.005$, respectively).

We further explored if specific complications occurred more frequently in specific age ranges within genders. In females, there was a higher occurrence of hypertension, diabetes, and osteoporosis in a range of 60-70 years. In males, hypertension was diagnosed a decade earlier than in females (in range 50-60). 
TABLE 1 | Prevalence of complications and their distribution in genders.

\begin{tabular}{|c|c|c|c|c|c|}
\hline Complication & $\begin{array}{c}\text { Number of } \\
\text { patients }\end{array}$ & $\%$ & F/M & $\% F$ & $\% \mathbf{M}$ \\
\hline Lipid disorders & 133 & 74 & $90 / 43$ & 76 & 72 \\
\hline Hypertension & 103 & 58 & $72 / 31$ & 61 & 52 \\
\hline Goiter & 93 & 52 & $65 / 28$ & 55 & 47 \\
\hline Joint degeneration & 72 & 40 & $46 / 26$ & 39 & 43 \\
\hline Hypopituitarism & 66 & 37 & $36 / 30$ & 30 & 50 \\
\hline Secondary hypogonadism & 33 & 18 & $13 / 20$ & 11 & 33 \\
\hline Secondary adrenal insufficiency & 46 & 26 & $25 / 21$ & 21 & 35 \\
\hline Secondary hypothyroidism & 40 & 22 & $21 / 19$ & 18 & 32 \\
\hline Panhypopituitarism & 18 & 10 & $6 / 12$ & 5 & 20 \\
\hline Changes in echocardiograms* & 61 & 34 & $36 / 25$ & 30 & 42 \\
\hline Prediabetes ${ }^{\star \star}$ & 61 & 34 & $33 / 28$ & 28 & 47 \\
\hline Diabetes ${ }^{\star \star \star}$ & 59 & 33 & $44 / 15$ & 37 & 25 \\
\hline Cholelithiasis & 51 & 28 & $36 / 15$ & 30 & 25 \\
\hline Arrhythmias & 35 & 20 & $20 / 15$ & 17 & 25 \\
\hline Osteoporosis & 22 & 12 & $15 / 7$ & 13 & 12 \\
\hline Nephrolithiasis & 22 & 12 & $13 / 9$ & 11 & 15 \\
\hline Colonic polyps & 21 & 12 & $13 / 8$ & 11 & 13 \\
\hline Ischemic heart disease & 12 & 7 & $11 / 1$ & 9 & 2 \\
\hline Heart failure & 11 & 6 & $6 / 5$ & 5 & 8 \\
\hline Carpal tunnel syndrome & 8 & 4 & $7 / 1$ & 6 & 2 \\
\hline Sleep apnea & 3 & 2 & $1 / 2$ & 1 & 3 \\
\hline
\end{tabular}

*Left ventricular and interventricular septum hypertrophy, diastolic dysfunction, valvular defects - mainly mitral valve regurgitation, improper atrial and ventricular dimensions.

${ }^{* *}$ Diagnostic criteria were: fasting plasma glucose between 100 and 125 mg/dL or twohour plasma glucose value between 140 and $199 \mathrm{mg} / \mathrm{dL}$.

${ }^{* * *}$ Diagnostic criteria were: fasting plasma glucose $\geq 126 \mathrm{mg} / \mathrm{dL}$ repeated twice or two-hour plasma glucose value of $\geq 200 \mathrm{mg} / \mathrm{dL}$ during a 75 -g oral glucose test (OGTT), or symptomatic hyperglycemia (weight loss, polyuria, polydipsia) and blood glucose $\geq 200 \mathrm{mg} / \mathrm{dL}$.

Additionally, we analyzed the prevalence of each complication according to a distribution of the acromegaly activity during the last hospitalization (Table 2). In order to analyze the coincidence of complications at a more general level, we aggregated complications into the three groups: metabolic, cardiovascular and endocrine (Table 3). The coincidence of the groups of complications revealed that $50.8 \%$ of patients suffered from at least one complication from each group. Frequency of co-occurrence of metabolic and cardiovascular disorders as well as metabolic and endocrine diseases was $15.5 \%$ for both. Only $5.0 \%$ of patients did not suffer from any disease belonging to those main groups.

Furthermore, an evaluation of the coexistence of particular complications by using heatmap clustering was performed (Figure 4). It revealed that types of pituitary insufficiency cooccurred more frequently. On this map, also a tendency to the coexistence of diabetes, hyperlipidemia, hypertension, and heart remodelling was observed. In addition, a cluster including prediabetes and cholelithiasis was obtained. Groups of diseases distinguished from the clustering are presented in Table 4.

\section{Analysis of Diagnostic Procedures}

We investigated the frequencies of diagnostic procedures performed or ordered during hospitalizations in the Endocrinology Department for diagnostics and monitoring purposes. They are listed in Table 5. The results of colonoscopies are presented in Table 6.

We analyzed the changes in numbers of procedures in 5-years periods. We have found that there was an increasing trend in the
TABLE 2 | Prevalence of complications and distribution of the disease activity during the last hospitalization.

\begin{tabular}{|c|c|c|c|c|}
\hline Complication & AA (\%) & WCA (\%) & SCA (\%) & Total \\
\hline Lipid disorders & $32(31)$ & $29(28)$ & $43(41)$ & 104 \\
\hline Hypertension & $29(30)$ & $34(35)$ & $34(35)$ & 97 \\
\hline Goiter & $20(24)$ & $36(43)$ & $28(33)$ & 84 \\
\hline Joint degeneration & $20(33)$ & $24(39)$ & $17(28)$ & 61 \\
\hline Hypopituitarism & 19 (33) & $19(33)$ & $20(34)$ & 58 \\
\hline Secondary hypogonadism & $9(33)$ & $8(30)$ & $10(37)$ & 27 \\
\hline Secondary adrenal insufficiency & $11(29)$ & $13(34)$ & $14(37)$ & 38 \\
\hline Secondary hypothyroidism & $12(32)$ & $12(32)$ & $13(35)$ & 37 \\
\hline Panhypopituitarism & $5(33)$ & $3(20)$ & $7(47)$ & 15 \\
\hline Changes in echocardiograms & $8(23)$ & $14(40)$ & $13(37)$ & 35 \\
\hline Prediabetes & $15(54)$ & $8(29)$ & $5(18)$ & 28 \\
\hline Diabetes & 18 (34) & $27(51)$ & $8(15)$ & 53 \\
\hline Cholelithiasis & $11(24)$ & $25(54)$ & $10(22)$ & 46 \\
\hline Arrhythmias & $10(50)$ & 7 (35) & $3(15)$ & 20 \\
\hline Osteoporosis & $6(32)$ & $6(32)$ & 7 (37) & 19 \\
\hline Nephrolithiasis & $6(38)$ & $3(19)$ & $7(44)$ & 16 \\
\hline Colonic polyps & $1(5)$ & $10(53)$ & $8(42)$ & 19 \\
\hline Ischemic heart disease & $2(25)$ & $5(63)$ & $1(13)$ & 8 \\
\hline Heart failure & $3(38)$ & $3(38)$ & $2(25)$ & 8 \\
\hline Carpal tunnel syndrome & $0(0)$ & $4(67)$ & $2(33)$ & 6 \\
\hline Sleep apnea & $0(0)$ & $2(67)$ & $1(33)$ & 3 \\
\hline
\end{tabular}

$A A$, active acromegaly; WCA, well-controlled acromegaly; SCA, surgery cured acromegaly.

number of performed thyroid ultrasounds $(\mathrm{p}=0.01)$. During this period, the number of performed abdomen ultrasounds correlated positively with the detectability of cholelithiasis $(p=0.02)$. There were no significant trends in other procedures.

\section{DISCUSSION}

Acromegaly complications are the main factors contributing to lifespan and its quality in patients, so active diagnostics and treatment are essential. The knowledge about comorbidities of acromegaly has changed in the past decades. For this moment, radical surgical treatment or even pharmacological control of the disease can enable reversibility of some complications, but only if those are recognized and treated in early stages. So, it is very important to shorten the delay in the diagnosis of acromegaly. In addition, it is essential to improve the regularity of diagnostic exams that help us to recognize comorbidities and treat them earlier. Obtaining a strict control of hormone excess is the best strategy to limit the development of complications of acromegaly.

\section{Patients Characteristics}

In the population of our patients, we can observe substantial domination of females. Similar outcomes were reported in Polish multicenter study (17), as well as in other European registries (18-23). Not statistically significant, but also a higher ratio of incidence of macroadenomas to microadenomas in men compared to women was detected in the French population (21).

As we expected, positive correlations between maximal tumor dimensions and IGF-I and GH concentrations were obtained in patients with an active phase of the disease. Similarly, such correlations were observed for acromegaly patients before 
TABLE 3 | Coincidence of complications belonging to the metabolic, cardiovascular and endocrine categories.

\begin{tabular}{lcccc}
\hline Metabolic & Cardiovascular & Endocrine & N & $\%$ \\
\hline 1 & 1 & 1 & 91 & 50.8 \\
1 & 1 & 0 & 28 & 15.6 \\
1 & 0 & 1 & 28 & 15.6 \\
1 & 0 & 0 & 11 & 6.1 \\
0 & 1 & 1 & 4 & 2.2 \\
0 & 0 & 1 & 8 & 4.5 \\
0 & 0 & 0 & 9 & 5.0
\end{tabular}

surgery (24). Adenoma measurements correlated positively with GH levels, but not with IGF-I levels in Tirosh et al. (25). and Evran et al. (26). studies, while Schwyzer et al. obtained contrary results with a significant correlation between preoperative tumor volume and IGF-I, but not with GH level (27). In Tirosh et al. study (25) an association between tumor volume and hypogonadism was determined, more pronounced in males, which was not obtained in our study. In that study also an association between size of tumor $>10 \mathrm{~mm}$ before the operation and the need for re-operation was observed. In our research, we also observed dependence between the maximal tumor diameter and reoperations in males, but in that analysis, we used data of maximal tumor diameter from all MRI results, also after an operation, not only before.

\section{Acromegaly Treatment}

Over the years, we observed a reduction in the number of patients with active acromegaly and an increase of pharmacologically controlled individuals. Consistent outcomes were depicted in the French registry (21). Many of the patients required additional treatment due to non-radical operation or recurrence. Sometimes decisions about reoperation are afflicted by difficult localization of the tumor, risk of damage to important structures (e.g. optic chiasm) or patients' coexisting diseases. Introduction and wide availability of somatostatin analogues allowed to achieve good control of disease activity and reduction in the occurrence of serious complications.

\section{Epidemiology and Pathogenesis of the Main Groups' Complications \\ Cardiovascular Complications}

Cardiovascular complications are the main causes of mortality in acromegaly patients $(2,3,8,13)$. Cure or even control of disease activity decrease the frequency and severity of comorbidities from this group. Some of them can even be totally reversed (28). In our study hypertension was the dominant complication from the cardiovascular group - it occurred in $58 \%$ of patients, which is consistent with previous studies. It has been reported that it occurs approximately in one-third of patients, ranging from 18 to $60 \%(4,17,28-30)$. What is more, the prevalence that we

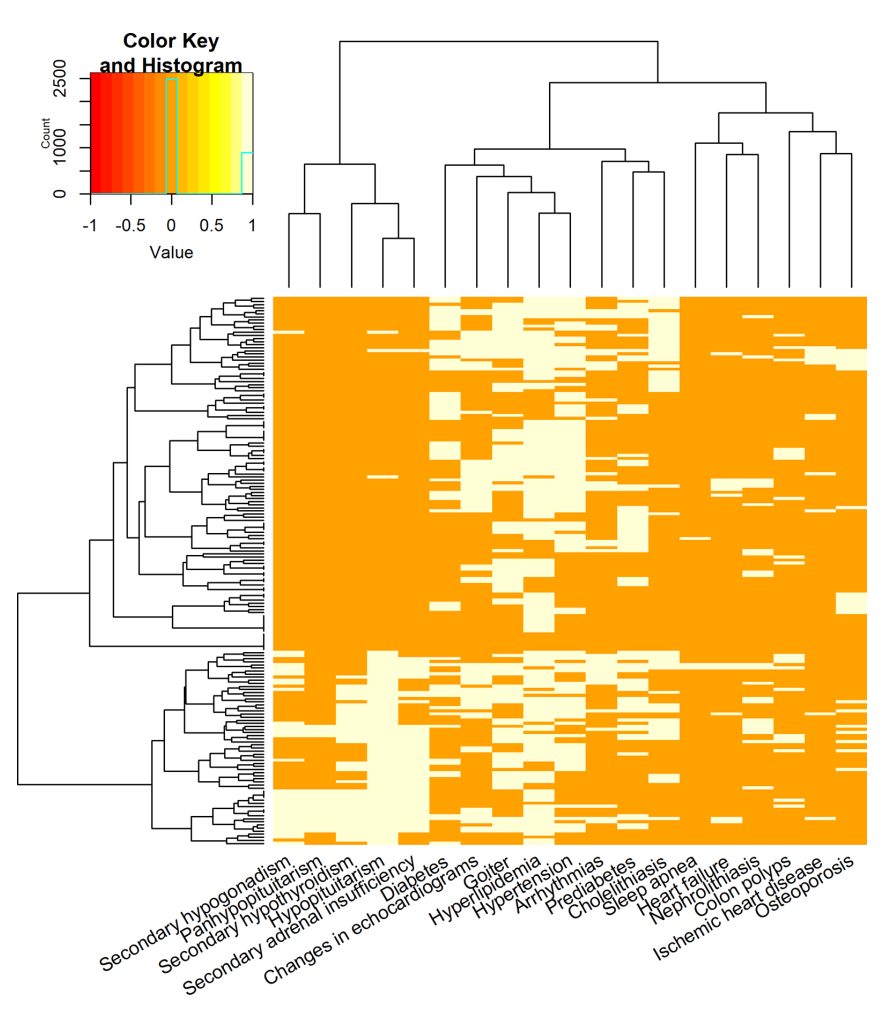

FIGURE 4 | Heatmap of particular complications co-occurrence. Each row in the heatmap denotes a patient. Each column is a complication. Bright fields mark complications that a patient suffered from. In addition, the dendrograms on the left-hand side and the top panel present grouping of patients, and grouping of complications, respectively. 
TABLE 4 | Groups of complications obtained from heatmap clustering.

I hypopituitarism, secondary hypogonadism, secondary hypothyroidism, secondary adrenal insufficiency, panhypopituitarism

II diabetes, hyperlipidemia, hypertension, changes in echocardiograms, goiter

III prediabetes, arrhythmias, cholelithiasis

IV osteoporosis, ischemic heart disease, heart failure, sleep apnea, colonic polyps, nephrolithiasis

TABLE 5 | Number of procedures performed during hospitalizations in the Endocrinology Department.

\begin{tabular}{lcc}
\hline Type of procedure & Number of procedures/496 hospitalizations & $\%$ \\
\hline BP measurement & 481 & 97.0 \\
Lipid profile & 462 & 93.1 \\
Fasting glucose & 453 & 91.3 \\
OGTT (if no diabetes) & 299 & 79.5 \\
Electrocardiogram & 391 & 78.8 \\
Densitometry & 235 & 47.4 \\
Hormonal profile* & 213 & 42.9 \\
Abdomen ultrasound & 224 & 45.2 \\
Thyroid ultrasound & 198 & 39.5 \\
Echocardiogram & 111 & 22.4 \\
Colonoscopy & 43 & 8.7
\end{tabular}

${ }^{*} F S H, L H, P R L, T S H, ~ f T 4$, morning cortisol, testosterone (in men), estradiol (in women).

TABLE 6 | The findings of colonoscopy in analyzed acromegaly patients.

\begin{tabular}{lc}
\hline Results & Number of patients \\
\hline Normal endoscopic appearance & 7 \\
Colonic polyps & 19 \\
Proximal colon to splenic flexure & 5 \\
Distal colon to splenic flexure & 9 \\
Proximal and distal colon & 3 \\
Rectum & 2 \\
Diverticulosis of the sigmoid colon & 5 \\
Grades Internal hemorrhoids & 14 \\
$\quad$ I & 6 \\
$\quad$ III & 5 \\
$\quad$ IV & 1 \\
External hemorrhoids & 2 \\
Colitis & 2 \\
Ulcerative colitis & 5 \\
Spastic colon & 1 \\
Irritable bowel syndrome (IBS) suspicion & 1 \\
\hline
\end{tabular}

I, II, III, IV: grades of internal hemorrhoids.

obtained is higher than in the general Polish population, which is $42.7 \%$ (31). The etiology of hypertension in acromegaly is multifactorial. It includes, inter alia: increased plasma volume, hypertrophy of vascular smooth muscles, vascular stiffness, and endothelial dysfunction $(28,29)$. Management of hypertension in acromegaly patients does not differ from that applied in the general population (14).

In other databases, left ventricle hypertrophy has been observed in $70-90 \%$ of reported cases [but only in $15 \%$ of patients in Liege Acromegaly Survey Database (19)] while valve diseases in 20 to $75 \%(4,28)$. In our study changes in echocardiograms (left ventricular and interventricular septum hypertrophy, diastolic dysfunction, valvular defects - mainly mitral valve regurgitation, improper atrial and ventricular dimensions) were found in $34 \%$ of the cases. The excess of $\mathrm{GH}$ and IGF-I is known to contribute to myocardial concentric hypertrophy, which leads to a decrease in the cardiac output and heart failure (29). We observed heart failure only in $6 \%$ of the cases, whereas the prevalence of this complication was previously described in approximately $10 \%$ of the cases (4). These differences may be a consequence of performing fewer examinations, including echocardiograms in the past.

There are conflicting data regarding acromegaly's contribution to a higher occurrence of ischemic heart disease (32). The probable origin of this complication is not associated with GH and IGF-I excess levels, but with other comorbidities like dyslipidemia, insulin resistance, hypertension, and sleep apnea (28). Ischemic heart disease was diagnosed in $7 \%$ of our patients. The incidence in previous registries was between 2.5 and $12 \%(17,19,28)$.

The most common arrhythmias observed in acromegaly are atrial fibrillation, sinus or ventricular tachycardia, sick sinus syndrome, and ventricular extrasystoles (28). Arrhythmias were reported to affect $7 \%$ to $48 \%$ of patients $(4,28)$. In our study, we observed them in $20 \%$ of the cases. The number of performed electrocardiograms was high (78.8\%), but it is a short time examination and it is possible that some disturbances might be detected only in Holter ECG.

\section{Metabolic Complications}

The prevalence of diabetes in acromegaly patients is $12 \%$ to $53 \%$ $(17,19,30,33,34)$. We observed diabetes in $33 \%$ of the cases and prediabetes in $34 \%$, which is much higher than in the general Polish population $(6.97 \%)$ (25). In Warncke's study, it has been reported that in acromegaly diabetes affects younger patients (50.1 years) than in the general population (59 years) (35). Comparable results were observed for the Italian population (20). In our research, the dominant age of diabetes diagnosis was 60 to 70 and 50 to 60 years for prediabetes, respectively. Factors that contribute to disturbances in glucose metabolism in acromegaly are complex (33). GH is an antagonist hormone to insulin, which increases lipolysis and gluconeogenesis and predisposes to insulin resistance, which is the main factor in diabetes origin $(33,36)$. Normalization of $\mathrm{GH}$ concentration decreases glycemia and improves insulin sensitivity (37). It is important to highlight that treatment with somatostatin analogues, especially pasireotide, may impede control of diabetes and increase insulin resistance $(13,38)$. Diabetes therapy in acromegaly does not differ from generally used in type 2 diabetes $(2,19)$.

Dyslipidemia, depending on the source, affects $13-51 \%$ or even up to $71 \%$ of acromegaly patients $(8,35)$. GH induces lipolysis and increases levels of plasma free fatty acids (8). Hypertriglyceridemia and decreased HDL level are the main lipid abnormalities in acromegaly $(8,36)$. In our study dyslipidemia was the most frequent complication in the whole group and it was observed in $74 \%$ cases, so it was even higher incidence compared to the previous studies $(8,35)$. Moreover, we observed elevated triglycerides in $48 \%$ cases, which is also higher in comparison to the other reports (4). 


\section{Endocrine Complications}

There is a proven correlation between thyroid gland volume and $\mathrm{GH}$ and IGF-I levels $(39,40)$. The prevalence of goiter in acromegaly patients reaches between 25 and $92 \%$ of the cases $(17,19,39,41,42)$. We observed this complication in $52 \%$ of our patients. Furthermore, a predisposition for the autonomous function of some nodules in nodular goiter contributes to hyperthyroidism (39). Some studies suggest a higher incidence of thyroid cancer in acromegaly $(39,43)$. In the presented series, we diagnosed one follicular thyroid cancer and one papillary thyroid cancer.

Hypopituitarism is caused by compression or damage of the pituitary gland by an expansion of adenoma or it is an iatrogenic complication that appears after surgery or radiotherapy. The prevalence of hypopituitarism in our study was $37 \%$ and it dominated in the male population. Similar prevalence of hormonal deficiency was reported in the Belgian registry (44). On the other hand, for example in the USA database, this complication was observed only in $16.6 \%$ of the cases (30). Secondary adrenal insufficiency was the most frequent alternation in our research. What is more, we observed hypopituitarism in $64.7 \%$ of patients after radiotherapy. It is known that radiotherapy increases the risk of hypopituitarism in this group it is diagnosed in more than half of the patients (36, 44 ). In contrary to the Italian registry (20), we observed the significant impact of radiotherapy only for hypogonadism in males. Hypopituitarism, secondary hypogonadism and panhypopituitarism were revealed more often in the male population. In the Italian registry, hypogonadism also predominated in males (20).

Acromegaly is also associated with secondary osteoporosis and fractures. Our research revealed osteoporosis in $12 \%$ of the cases. We did not have complete information about fractures in the analyzed group of patients. In previous studies, osteoporosis was observed in $12-32 \%$ of patients $(19,45)$. It is known that a higher risk of fractures may occur despite normal bone mineral density (BMD) (46). So, we still need new tools to estimate the risk of fractures in acromegaly. Assessment of bone quality can improve this evaluation. Recently introduced trabecular bone score (TBS) method is a promising tool, which enables to assess bone tissue microarchitecture $(47,48)$.

\section{Coexistence of Complications}

In more than half of patients, we observed co-occurrence of cardiovascular, metabolic and endocrine complications. This finding illustrates that they are common problems in acromegaly and acromegaly patients often require complex healthcare and multi-component therapy. Cardiovascular and metabolic, as well as metabolic and endocrine complications co-occurred more frequent than cardiovascular and endocrine disorders. The coincidence of hypertension, heart structural changes, hyperlipidemia and diabetes was also exemplified in heatmap clustering evaluation. Besides the impact of GH and IGF-I excess on cardiovascular and metabolic diseases, similar environmental factors contribute to the origin of morbidities classified in those groups. We speculate that obtained cluster of prediabetes and cholelithiasis coexistence may indicate on the group of patients treated with somatostatin analogues. Moreover, in the analysis of disease activity (Table 2), diabetes and cholelithiasis were observed in more than $50 \%$ in the well-controlled acromegaly patients treated with somatostatin analogues.

\section{Diagnostics of Complications}

We decided to determine if patients from our department underwent procedures that are recommended in the guidelines. According to current worldwide and Polish guidelines $(14,49)$ BP measurement, fasting glucose or OGTT should be performed every six months; ECG, echocardiography, Epworth scale, spine $\mathrm{X}$-ray, lipid and hormonal profile (towards hypopituitarism diagnostics) and Acromegaly Quality of Life Questionnaire (AcroQoL) annually; thyroid ultrasound every 1 to 2 years; DXA every 2 years and colonoscopy every 10 years. In order to do that we calculated the general number of each procedure during 496 hospitalizations (Table 5). According to obtained results BP measurement, ECG, lipid profile, fasting glucose, or OGTT were performed the most often. The least frequent examinations were colonoscopy and echocardiogram. None of our patients had polysomnography. This might be a result of the clinic profile. Polysomnography, colonoscopy and echocardiogram require the presence of other specialists to be performed. Unrecognized sleep apnea, colon cancer or heart failure can lead to serious consequences. It is important to highlight that in care of acromegaly patients, the involvement of different types of specialists is needed and that each patient should obtain referrals for additional consultations when needed. In 21 patients (12\%) (in 19 cases colonoscopy was recommended in the Endocrinology Department, in 2 an information about diagnosis was included in the medical histories) colonic polyps were observed. Colonic polyps are the most frequent types of tumors in acromegaly (39), so obtained rate indicates that this diagnosis could be underestimated in the group of our patients, but it is similar to observed in the Liege Acromegaly Survey Database (19). Hemorrhoids and diverticulosis, which also are reported to be associated with GH and IGF-I excess (4), were detected in 37 and $12 \%$ of examinations, respectively. The number of performed abdomen ultrasounds correlated positively with cholelithiasis detectability. Cholelithiasis might be a side effect of somatostatin analogues treatment, thus ultrasound is recommended, especially in pharmacologically treated group. In addition, the medical histories lack the information regarding the performance of AcroQoL, thus we speculate that it has not been conducted. Nowadays recommendations and studies emphasize that life quality assessment is very important in the evaluation of the effectiveness of treatment in acromegaly $(10,11,14)$.

\section{CONCLUSIONS}

In our population we observed female predominance. Over the years we noticed a reduction in the number of patients with active acromegaly and an increase of patients with 
pharmacologically controlled disease. More than $50 \%$ of patients demonstrated a coexistence of cardiac, metabolic and endocrine disturbances and what is important to decline only $5 \%$ of patients did not suffer from any disease from those main groups. For these reasons, an obtain a strict control of hormone excess is the best strategy to limit the development of complications of acromegaly.

\section{DATA AVAILABILITY STATEMENT}

The original contributions presented in the study are included in the article, further inquiries can be directed to the corresponding author.

\section{ETHICS STATEMENT}

The studies involving human participants were reviewed and approved by Bioethics Committee of the Wroclaw Medical University, Wrocław, Poland. Written informed consent for

\section{REFERENCES}

1. Melmed S, Bronstein MD, Chanson P, Klibanski A, Casanueva FF, Wass JAH, et al. A Consensus Statement on acromegaly therapeutic outcomes. Nat Rev Endocrinol (2018) 14:552-61. doi: 10.1038/s41574-018-0058-5

2. Abreu A, Tovar AP, Castellanos R, Valenzuela A, Giraldo CMG, Pinedo AC, et al. Challenges in the diagnosis and management of acromegaly: a focus on comorbidities. Pituitary (2016) 19:448-57. doi: 10.1007/s11102-016-0725-2

3. Giustina A, Casanueva FF, Cavagnini F, Chanson P, Clemmons D, Frohman LA, et al. Diagnosis and treatment of acromegaly complications. J Endocrinol Invest (2003) 26:1242-7. doi: 10.1007/BF03349164

4. Pivonello R, Auriemma RS, Grasso LF, Pivonello C, Simeoli C, Patalano R, et al. Complications of acromegaly: cardiovascular, respiratory and metabolic comorbidities. Pituitary (2017) 20:46-62. doi: 10.1007/s11102-017-0797-7

5. Sherlock M, Ayuk J, Tomlinson JW, Toogood AA, Aragon-Alonso A, Sheppard MC, et al. Mortality in patients with pituitary disease. Endocr Rev (2010) 31:301-42. doi: 10.1210/er.2009-0033

6. Ritvonen E, Löyttyniemi E, Jaatinen P, Ebeling T, Moilanen L, Nuutila P, et al. Mortality in acromegaly: A 20-year follow-up study. Endocr Relat Cancer (2016) 23:469-80. doi: 10.1530/ERC-16-0106

7. Kasuki L, Rocha PDS, Lamback EB, Gadelha MR. Determinants of morbidities and mortality in acromegaly. Arch Endocrinol Metab (2019) 63:630-7. doi: 10.20945/2359-3997000000193

8. Gadelha MR, Kasuki L, Lim DST, Fleseriu M. Systemic complications of acromegaly and the impact of the current treatment landscape: an update. Endocr Rev (2019) 40:268-322. doi: 10.1210/er.2018-00115

9. Geraedts VJ, Andela CD, Stalla GK, Pereira AM, Van Furth WR, Sievers C, et al. Predictors of quality of life in acromegaly: No consensus on biochemical parameters. Front Endocrinol (2017) 8:40:40. doi: 10.3389/fendo.2017.00040

10. Jawiarczyk-Przybyłowska A, Szcześniak D, Ciułkowicz M, Bolanowski M, Rymaszewska J. Importance of illness acceptance among other factors affecting quality of life in acromegaly. Front Endocrinol (2020) 10:899:899. doi: 10.3389/fendo.2019.00899

11. Tseng FY, Chen ST, Chen JF, Huang TS, Lin JD, Wang PW, et al. Correlations of clinical parameters with quality of life in patients with acromegaly: Taiwan Acromegaly Registry. J Formos Med Assoc (2019) 118:1488-93. doi: 10.1016/ j.jfma.2019.05.007

12. Mazziotti G, Bianchi A, Porcelli T, Mormando M, Maffezzoni F, Cristiano A, et al. Vertebral fractures in patients with acromegaly: A 3-year prospective study. J Clin Endocrinol Metab (2013) 98:3402-10. doi: 10.1210/jc.2013-1460 participation was not required for this study in accordance with the national legislation and the institutional requirements.

\section{AUTHOR CONTRIBUTIONS}

MR, AJ-P, and MB contributed to the study conception and design. Material preparation and data collection were performed by MR, GZ, AJ-P, JH-Ż, MK, and IB. MR created the database. BK performed the statistical analysis. MR, AJ-P, JH-Ż, and BK interpreted the results. The first draft of the manuscript was written by MR and AJ-P and corrected by JH-Ż, BK, GZ, and MB. All authors contributed to the article and approved the submitted version.

\section{FUNDING}

This study was supported by Statutory Activities by the Minister of Science and Higher Education (grant number SUB.C120.21.025).

13. Melmed S, Casanueva FF, Klibanski A, Bronstein MD, Chanson P, Lamberts SW, et al. A consensus on the diagnosis and treatment of acromegaly complications. Pituitary (2013) 16:294-302. doi: 10.1007/s11102-012-0420-x

14. Giustina A, Barkan A, Beckers A, Biermasz N, Biller BMK, Boguszewski C, et al. A consensus on the diagnosis and treatment of acromegaly comorbidities: An update. J Clin Endocrinol Metab (2020) 105:e937-947. doi: 10.1210/clinem/dgz096

15. Katznelson L, Laws ERJr, Melmed S, Molitch ME, Murad MH, Utz A, et al. Acromegaly: An Endocrine Society Clinical Practice Guideline. J Clin Endocrinol Metab (2014) 99:3933-51. doi: 10.1210/jc.2014-2700

16. Murtagh F, Legendre P. Ward's hierarchical agglomerative clustering method: which algorithms implement Ward's criterion? J Classif (2014) 31:274-95. doi: 10.1007/s00357-014-9161-Z

17. Bolanowski M, Zgliczyński W, Sowiński J, Bałdys-Waligórska A, BednarekTupikowska G, Witek P, et al. Therapeutic effect of presurgical treatment with long- acting octreotide (Sandostatin ${ }^{\circledR}$ LAR $^{\circledR}$ ) in patients with acromegaly. Endokrynol Pol (2020) 71:285-91. doi: 10.5603/EP.a2020.0050

18. Kamusheva M, Vandeva S, Mitov K, Rusenova Y, Elenkova A, Zacharieva S, et al. New epidemiological, clinical and economic data for patients with acromegaly in Bulgaria. Front Public Heal (2020) 8:147:147. doi: 10.3389/ fpubh.2020.00147

19. Petrossians P, Daly AF, Natchev E, Maione L, Blijdorp K, Sahnoun-Fathallah $\mathrm{M}$, et al. Acromegaly at diagnosis in 3173 patients from the Liège Acromegaly Survey (LAS) Database. Endocr Relat Cancer (2017) 24:505-18. doi: 10.1530/ ERC-17-0253

20. Arosio M, Reimondo G, Malchiodi E, Berchialla P, Borraccino A, De Marinis L, et al. Predictors of morbidity and mortality in acromegaly: an Italian survey. Eur J Endocrinol (2012) 167:189-98. doi: 10.1530/EJE-12-0084

21. Maione L, Brue T, Beckers A, Delemer B, Petrossians P, Borson-Chazot F, et al. Changes in the management and comorbidities of acromegaly over three decades: the French Acromegaly Registry. Eur J Endocrinol (2017) 176:64555. doi: 10.1530/EJE-16-1064

22. Sesmilo G. Epidemiology of acromegaly in Spain. Endocrinol Nutr (2013) 60:470-4. doi: 10.1016/j.endonu.2012.09.010

23. Schöfl C, Franz H, Grussendorf M, Honegger J, Jaursch-Hancke C, Mayr B, et al. Long-term outcome in patients with acromegaly: analysis of 1344 patients from the German Acromegaly Register. Eur J Endocrinol (2012) 168:39-47. doi: 10.1530/EJE-12-0602

24. Cardinal T, Rutkowski MJ, Micko A, Shiroishi M, Jason Liu CS, Wrobel B, et al. Impact of tumor characteristics and pre- and postoperative hormone 
levels on hormonal remission following endoscopic transsphenoidal surgery in patients with acromegaly. Neurosurg Focus (2020) 48:E10. doi: 10.3171/ 2020.3.FOCUS2080

25. Tirosh A, Papadakis GZ, Chittiboina P, Lyssikatos C, Belyavskaya E, Keil M, et al. 3D volumetric measurements of GH secreting adenomas correlate with baseline pituitary function, initial surgery success rate, and disease control. Horm Metab Res (2017) 49:440-5. doi: 10.1055/s-0043-107245

26. Evran M, Sert M, Tetiker T. Clinical experiences and success rates of acromegaly treatment: the single center results of 62 patients. BMC Endocr Disord (2014) 14:97. doi: 10.1186/1472-6823-14-97

27. Schwyzer L, Starke RM, Jane JAJr, Oldfield EH. Percent reduction of growth hormone levels correlates closely with percent resected tumor volume in acromegaly. J Neurosurg (2015) 122:798-802. doi: 10.3171/2014.10.JNS14496

28. Ramos-Leví AM, Marazuela M. Cardiovascular comorbidities in acromegaly: an update on their diagnosis and management. Endocrine (2017) 55:346-59. doi: 10.1007/s12020-016-1191-3

29. Isgaard J, Arcopinto M, Karason K, Cittadini A. GH and the cardiovascular system: an update on a topic at heart. Endocrine (2015) 48:25-35. doi: 10.1007/ s12020-014-0327-6

30. Broder MS, Neary MP, Chang E, Cherepanov D, Katznelson L. Treatments, complications, and healthcare utilization associated with acromegaly: a study in two large United States databases. Pituitary (2014) 17:333-41. doi: 10.1007/ s11102-013-0506-0

31. Niklas A, Flotyńska A, Puch-Walczak A, Polakowska M, Topór-Mądry R, Polak M, et al. Prevalence, awareness, treatment and control of hypertension in the adult Polish population - Multi-center National Population Health Examination Surveys - WOBASZ studies. Arch Med Sci (2018) 14:951-61. doi: 10.5114/aoms.2017.72423

32. Sharma MD, Nguyen AV, Brown S, Robbins RJ. Cardiovascular disease in acromegaly. Methodist Debakey Cardiovasc J (2017) 13:64-7. doi: 10.14797/mdcj$13-2-64$

33. Hannon AM, Thompson CJ, Sherlock M. Diabetes in patients with acromegaly. Curr Diabetes Rep (2017) 17:8. doi: 10.1007/s11892-017-0838-7

34. Mercado M, Ramírez-Rentería C. Metabolic complications of acromegaly. Front Horm Res (2018) 49:20-8. doi: 10.1159/000486001

35. Warncke K, Kummer S, Kann PH, Bergis D, Bollow E, Hummel M, et al. Cardivascular risk profile in patients with diabetes and acromegaly or Cushing's disease - Analysis from the DPV database. Exp Clin Endocrinol Diabetes (2020) 128:104-10. doi: 10.1055/a-0600-9649

36. Colao A, Ferone D, Marzullo P, Lombardi G. Systemic complications of acromegaly: Epidemiology, pathogenesis, and management. Endocr Rev (2004) 25:102-52. doi: 10.1210/er.2002-0022

37. Møller N, Jørgensen JO. Effects of growth hormone on glucose, lipid, and protein metabolism in human subjects. Endocr Rev (2009) 30:152-77. doi: 10.1210/er.2008-0027

38. Shimon I, Adnan Z, Gorshtein A, Baraf L, Saba Khazen N, Gershinsky M, et al. Efficacy and safety of long-acting pasireotide in patients with somatostatinresistant acromegaly: a multicenter study. Endocrine (2018) 62:448-55. doi: $10.1007 / \mathrm{s} 12020-018-1690-5$
39. Tirosh A, Shimon I. Complications of acromegaly: thyroid and colon. Pituitary (2017) 20:70-5. doi: 10.1007/s11102-016-0744-Z

40. Yang H, Zhang MZ, Wei J, Chen J, Yu YR, An ZM, et al. Risk Factors Associated with Thyroid Nodules in Patients with Acromegaly. Sichuan Da Xue Xue Bao Yi Xue Ban (2019) 50:433-7.

41. Gasperi M, Martino E, Manetti L, Arosio M, Porretti S, Faglia G, et al. Prevalence of thyroid diseases in patients with acromegaly: Results of an Italian multi-center study. J Endocrinol Invest (2002) 25:240-45. doi: 10.1007/ BF03343997

42. Natchev E, Vandeva S, Kovatcheva R, Kirilov G, Kalinov K, Zacharieva S. Thyroid gland changes in patients with acromegaly. Arch Endocrinol Metab (2020) 64:269-75. doi: 10.20945/2359-3997000000247

43. Wolinski K, Czarnywojtek A, Ruchala M. Risk of thyroid nodular disease and thyroid cancer in patients with acromegaly - Meta-analysis and systematic review. PloS One (2014) 9:e88787. doi: 10.1371/ journal.pone. 0088787

44. Bex M, Abs R, T'Sjoen G, Mockel J, Velkeniers B, Muermans K, et al. AcroBel the Belgian registry on acromegaly: a survey of the 'real-life' outcome in 418 acromegalic subjects. Eur J Endocrinol (2007) 157:399-409. doi: 10.1530/EJE07-0358

45. Padova G, Borzì G, Incorvaia L, Siciliano G, Migliorino V, Vetri M, et al. Prevalence of osteoporosis and vertebral fractures in acromegalic patients. Clin Cases Miner Bone Metab (2011) 8:37-43.

46. Mazziotti G, Frara S, Giustina A. Pituitary diseases and bone. Endocr Rev (2018) 39:440-88. doi: 10.1210/er.2018-00005

47. Hong AR, Kim JH, Kim SW, Kim SY, Shin CS. Trabecular bone score as a skeletal fragility index in acromegaly patients. Osteoporos Int (2016) 27:11239. doi: 10.1007/s00198-015-3344-2

48. Jawiarczyk-Przybyłowska A, Halupczok-Żyła J, Kolačkov K, Gojny Ł, Zembska A, Bolanowski M. Association of vitamin D receptor polymorphisms with activity of acromegaly, vitamin D status and risk of osteoporotic fractures in acromegaly patients. Front Endocrinol (2019) 10:643:643. doi: 10.3389/fendo.2019.00643

49. Bolanowski M, Ruchała M, Zgliczyński W, Kos-Kudła B, HubalewskaDydejczyk A, Lewiński A. Diagnostics and treatment of acromegaly updated recommendations of the Polish Society of Endocrinology. Endokrynol Pol (2019) 70:2-18. doi: 10.5603/EP.a2018.0093

Conflict of Interest: The authors declare that the research was conducted in the absence of any commercial or financial relationships that could be construed as a potential conflict of interest.

Copyright (C) 2021 Rolla, Jawiarczyk-Przybyłowska, Halupczok-Żyła, Kałużny, Konopka, Btoniecka, Zielinski and Bolanowski. This is an open-access article distributed under the terms of the Creative Commons Attribution License (CC BY). The use, distribution or reproduction in other forums is permitted, provided the original author(s) and the copyright owner(s) are credited and that the original publication in this journal is cited, in accordance with accepted academic practice. No use, distribution or reproduction is permitted which does not comply with these terms. 